\title{
Convergence of Smart Technologies for Digital Transformation
}

\author{
Bertrand Mareschal, Mandeep Kaur, Vilas Kharat, Sachin Sakhare
}

\begin{abstract}
This foreword is related to the sixteen papers published in this issue, which were presented at IC2ST-2021 - International Conference on Convergence of Smart Technologies. The Aspire Research Foundation organized this conference in Pune, India, January 9-10, 2021. These sixteen papers have not and will not be published anywhere else.
\end{abstract}

Keywords: Digital Transformation; Smart Technologies

\section{Preface}

The convergences of smart technologies have brought digital revolution with a paradigm shift from manual operations to automated operations in the world around us. The convergence of technologies is playing a fundamental role in the growth and evolution of smart and embedded systems in this digital era. We can imagine a world where robots, sensors, automated guided vehicles (AGVs), products, databases communicate and controllers with one another. The smart systems assimilate elements of computing with the physical processes and components. The computing elements communicate with sensors and these sensors monitor physical indicators, which adapt the cyber-physical environment. The convergence of technologies enable the cyber-physical systems (CPSs) to make use of sensors to connect the distributed intelligence for gaining greater knowledge of the environment and for taking more accurate actions and tasks. The design of smart systems for industry 4.0 and future requires joint dynamics of computers, software, networks, and physical processes.

The smart technologies comprise distributed systems, communication systems, computational resources, data resources and control components connected with diverse physical processes. However, the fundamental design requirement for smart systems is that the hardware components, cyber components, distributed systems, communication technologies and computational resources should be able to work altogether to deliver the expected functionality accurately.

The combination of the smart technologies, cyber physical systems, Internet of Things, artificial intelligence and embedded systems will drive innovation and effectiveness in a global marketplace. The convergence of smart technologies will enable us to gather and analyze big data across smart devices and will assist faster, scalable, and efficient processes to produce better-quality goods at the cheapest costs.

\section{The Issues to Be Addressed for the Successful Implementation of Smart Technologies}

\section{- Security}

The most important concern for businesses is security as they embark the 4.0 journey. This includes both cyber and physical security. The industries are worried about the followings:

- Stealing of Intellectual Property Rights

- Stealing of data containing client information

- Hacking of Machines for ransom

The workstations and other systems are at higher risk as the attack surface is increasing day by day and it is easy to attack by using malwares, adwares and phishing scams. The hackers are not only targeting industrial systems but also targeting the IoT-cloud collaborative environments where several users are connected through diverse devices. Usage of smart technologies has introduced new threats to users and proprietary data.

\section{- Adoption}

The next challenging task for smart technologies is adoption. An automation and evolution of robotics technology is posing a potential threat to the present workforce, which will be replaced by machines, and this issue has to be addressed carefully. The workforce reduction will be resultant from the evolution of smart technologies but it may lead to unemployment around the world. Industry 4.0 may need very skilled workforce. The developing and underdeveloped countries may be reluctant to adapt the smart technologies to avoid the situation of unemployment and resistance from the current workforce. If the skilled workforce will be required then it may not be possible to train the existing workforce but new workforce can be trained where young generation can certainly contribute. This will drive a solution for the front-line production and second-line functional support. The adaption of latest technologies will remain questionable with respect to adaption of these technologies at global level. 


\section{- Seamless Connectivity}

Connecting workstations, laptops, mobile phones, IoT devices, deploying IoT sensors, through seamless communication channels and collaboration tools will drive a need for highly reliable and secure internet. People around the world will not be able to take advantage of the smart technologies until there is a promising seamless connectivity solutions delivered by the researchers and industrialists.

\section{- Standardization}

Implementing new techniques, deployment of smart systems will need many disparate technologies to connect and communicate. There is a need to set some standards over the existing standards that can promise seamless connectivity, smooth communication and AI based automated solutions. Industry 4.0 and smart world cannot be created overnight, there are interoperability needs and standard solutions to be developed to permit data from one global position to another global position irrespective of diverse underlying architectures, gateways and transmission media. This implies inclusion of smart technologies in the present world will be a long-term evolution. The standards should be introduced, implemented and accepted worldwide.

\section{The Pillars of Smart Technologies}

\section{- Internet of Things (IoT)}

The physical world has been transformed into the digital world where everything is connected seamlessly. An eruption of IoT devices and latest technologies has permitted humanbeings to stay connected irrespective of geographical locations. Internet of Things has generated a sub-segment as industrial Internet of Things, which is known as IIoT. Industry 4.0 with IIoT is anticipated to transform the production line in industries, the way we live, the way we work and the way we interact with the digitized world.

\section{- Big Data}

The greatest challenge for IIoT is privacy and security. IoT and the seamless connectivity means gathering of real time data from multiple places for enabling quick decisions to enhance productivity and efficiency. The key advantage of IIoT is the data it generates and transforms the data into actionable insights.

\section{- $\quad$ Digital Twin}

Digital twins (DTs) are gaining extensive consideration from industry engineers, researchers and academicians. Cyber physical systems and digital twins can impact manufacturing and production systems with greater efficacy, intelligence and resilience. There are many revolutionary digital technologies evolving to gear up and restructure the activities to fulfil digital transformation objectives. Digital twin comprises technologies for the upcoming years of automated, knowledge-filled, goal-oriented and humancentric services.

\section{- Cyber Physical Systems}

Cyber-Physical Systems are assimilations of embedded systems, computation, physical processes and networking. Embedded systems and computer networks control and monitor the physical processes, where physical processes use computations and form the base for futuristic smart devices to improve quality of life in diverse areas. Cyber-physical systems will improve health care systems, emergency services, management of traffic flow, service sector, manufacturing and production line in industries, and many other areas where computer intelligence can be applied. Cyber-Physical Systems will provide the basis of critical infrastructure, and futuristic intelligent services.

\section{- Cloud Computing}

Cloud computing provides on-demand computational and data-storage resources to the users. Instead of purchasing, possessing, and maintaining data storage centers and servers, the industries can access cloud technology services such as computational power, software instances and data-storage space on as-and-when required basis from the cloud providers. Industries irrespective of size are using the cloud services such as data backup, email, disaster recovery, software development, big data handling, and web based applications. Cloud computing is one of the most important building blocks of smart world as it will help the world with easy access to a wide-ranging of technologies. With the cloud, industries can grow to new geographic areas and deploy internationally in minutes.

\section{- Artificial Intelligence}

The progressive adoption of Artificial intelligence and machine learning techniques improve the flow of resources through the manufacturing process. In industry 4.0 scenario, an AI becomes an essential factor that assists enterprises in digitization of the manufacturing sector. We can also say that $\mathrm{AI}$ is the driving force for this century. AI can assist the world in predicting maintenance, generative designing, manufacturing, delivering quality, human-robot collaboration, supply-chain management, waste reduction, and in production optimization.

\section{- Simulation}

Nowadays industries are able to manufacture things in a more fascinating way by using simulations. We can say that innovative technologies and artificial intelligence based systems have given a fair share for producing simulations based products in today's era. In simulation, the computer models are capable enough to simulate the operations of any real world system. Perhaps the appropriate simulated systems can assist an organization is to approximation of the return on investment before the product is actually initiated.

\section{- Cybersecurity}

Smart and intelligent systems require security along with preserving the ideas of scalability and functionality. IoT security solutions must be introduced where all the devices are connected and information can be shared seamlessly. Cybersecurity attacks are increasing more frequently day by 
day as attackers exploit vulnerabilities of the systems for financial gain. Cyber attackers have security controls and are attempting to improve their attacking techniques. In order to use the smart technologies optimally, it is important to impose strong cybersecurity controls over the systems.

Digital transformation with the evolution of smart technologies is the profound transformation of the businesses, organizational activities, processes, competencies and models. In order to fully leverage the changes and opportunities of the smart digital technologies and their accelerating impact across society in a strategic way, there is a need to handle the aforementioned issues and to strengthen the pillars of smart technologies. The smart technologies should be embraced by the society and the business world globally to create the capabilities of fully leveraged possibilities \& opportunities of new technologies and their impact in a faster, better and in an innovative way in the future.

\section{Authors' contacts:}

Dr. Bertrand Mareschal, Professor

Solvay Brussels School of Economics \& Management, Avenue F. D. Roosevelt 50, 1050, Brussels, Belgium

bmaresc@ulb.ac.be

Dr. Mandeep Kaur, Managing Director

Aspire Research Foundation,

404/405, Pinnacle Prestige, Tilak Road, Pune, India

mandeep.kaur@aspireresearch.in

Dr. Vilas Kharat, Professor

Dept. of Mathematics, Savitribai Phule Pune University,

Pune - 411 007, India

vilaskharat@unipune.ac.in

Dr. Sachin S. Sakhare, Professor and Head

Dept. of CSE, Vishwakarma Institute of Information Technology,

Bibwewadi, Pune - 411037, Maharashtra, India

sachin.sakhare@viit.ac.in 